\title{
Non Contiguous Tri-band Receiver for Carrier Aggregation RF System
}

\author{
Kaissoine Abdou, Bernard Huyart* \\ Department of Communications and Electronics, TELECOM ParisTech, France
}

Copyright $\bigcirc 2016$ by authors, all rights reserved. Authors agree that this article remains permanently open access under the terms of the Creative Commons Attribution License 4.0 International License

\begin{abstract}
The carrier aggregation technique will allow an increase of capacity of the $4^{\text {th }}$ Generation mobile communication networks (LTE-A: Long Term Evolution Advanced). The probability to find carriers that occupy a continuous spectrum in frequency is very low. The challenge is to demodulate an RF signal where the information is distributed on non-contiguous frequency bands. The technique presented in this paper concerns the mixing of $n$ modulated carriers with n Continuous Wave (CW) signals by using a single three phase demodulator circuit. This technique allows reducing the conversion band of the Analog to Digital Converter (ADC) to $100 \mathrm{MHz}$. The principle is theoretically demonstrated for three non-contiguous carriers and validated by measurements results. The test was made for modulated carriers of $10 \mathrm{MHz}$ or $15 \mathrm{MHz}$ bandwidth by using the same QPSK modulation format or two different modulation formats (QPSK and 16-QAM). The demodulation performance is evaluated by measuring the Error Vector Magnitudes (EVM) of the transposed signals in baseband. This test shows that it is possible to demodulate a distributed non-contiguous multi-carriers RF signal in the band 2-3 GHz with a bit rate more than $300 \mathrm{Mbps}$.
\end{abstract}

Keywords Spectrum Aggregation, Down Converter, RF Receiver, LTE-advanced, Three Phase Demodulator, Error Vector Magnitude, Low-IF, Sources Synchronization

\section{Introduction}

According to the International Telecommunication Union, the $4^{\text {th }}$ generation of mobile networks (LTE-Advanced or IMT-Advanced) can reach a theoretical data rate of $3 \mathrm{Gbps}$ (in downlink) $[1,3]$. To achieve very high data rates, it is necessary to increase the width of the transmission bandwidth. The proposed method is called carrier aggregation $[1,2]$. The carriers can be distributed in a contiguous or non-contiguous manner [3]. In the frequency band from 0.45 to $3.6 \mathrm{GHz}$, the available frequency sub-bands are non-contiguous and often as small as $20 \mathrm{MHz}$ [4]. We must, therefore, aggregate five non-contiguous frequency bands if we want to get the $100 \mathrm{MHz}$ of bandwidth needed to reach the desired data rate. The present technical challenge is to demodulate a RF signal whose information is centered on non-contiguous multi-carriers.

Demodulation consists generally to mix the RF signal with a local oscillator (LO) signal composed of a single $\mathrm{CW}$ signal. In the case where non-contiguous RF bands are distributed over a wide spectrum, the band of the intermediate frequency signal can be very large $(3 \mathrm{GHz})$, which requires an ADC sampling frequency of at least $6 \mathrm{GHz}$. The technique proposed in this paper consists in the RF signal mixing with an LO signal composed of $\mathrm{n} \mathrm{CW}$ signals which will have the main advantage that the intermediate frequency signal will cover a spectrum of 0 to $100 \mathrm{MHz}$ and reduce the ADC sampling frequency.

The existing solutions in the literature are often limited to two frequency bands. Among them, there is a solution that uses a similar technique than the demodulator architecture of type Weaver $[5,6]$ which rejects the image signal and keeps the desired signal. The architecture uses a single frequency for the LO signal to transpose two RF signals into baseband; By choosing correctly the value of the LO frequency, we may be able to correspond correctly the desired and image signals to the double frequency aggregated bands; The LO frequency is approximately equal to the half of two carrier frequencies of the non-contiguous frequency bands. Weaver architecture is not applicable to the case of three aggregated bands. [7] also describes a technique that allows to demodulate two non-contiguous frequency bands, by using a single LO signal which is somewhat close to Weaver architecture. It uses a single frequency for the LO signal but with a classic IQ demodulator. The two aggregated signals are transposed in two intermediate frequencies (IF). These two baseband signals are symmetrical relative to zero frequency i.e. one is the image of the other. This solution is not, also, applicable to a RF signal where the information is distributed over two non-contiguous carrier frequencies. Another technique is presented in the literature [8-10]: It 
uses two classic IQ demodulators to simultaneously transpose both non-contiguous frequency bands into baseband. In the case of five frequency bands, it should require five receivers, which makes this type of architecture onerous.

In parallel of our research works, a team of NTT (Nippon Telegraph and Telephone) published in [11], measurements results on the baseband transposition by mixing of a RF signal composed of two non-contiguous frequency bands with a LO signal constituted of two $\mathrm{CW}$ tones. The technique of conversion in low frequency signals by a single mixer is made each time by controlling the power level of one of the two CW signals. Another technique close to that of [11] is presented in [12]. This paper shows that it is possible to demodulate a dual-band RF signal, by using a LO signal that is the combination of two $\mathrm{CW}$ signals with a unique multiport demodulator ( $\mathrm{Rx}$ six-port).The six-port receiver uses power detectors for converting the baseband signals. This work presented measurement results of the demodulation of two modulated signals in 64-QAM and 16-QAM (64 and 16-states Quadrature Amplitude Modulation) with a bandwidth of $2 \mathrm{MHz}$, each one. The two signals are converted in low frequency in ZIF (Zero Intermediate Frequency) and LIF (Low Intermediate Frequency) centered at $8 \mathrm{MHz}$, respectively. The main disadvantage of the multiport demodulator circuits (five-port or six-port) is the low sensitivity due to the high conversion losses of the power detectors [13]. In figure 7 of [12], the measured spectrum at the output of the power detector has a power level of $-43 \mathrm{dBm}$. The measurement is done at a RF input power level equal to $0 \mathrm{dBm}$. This result confirms that the power detectors have a very low sensitivity. For example in [13], the measurements show that at $2.1 \mathrm{GHz}$, the conversion losses for power detector of Schottky HSMS-2850 diode is almost $39 \mathrm{~dB}$. The solution of [12] is similar to that one of $[14,15]$. The developed technique allows down-conversion of multiple frequency aggregated signals, by using a single multiport demodulator circuit, named "Three Phase Demodulator (TPD)". The TPD has a low conversion loss (in the case of passive mixers), see even a conversion gain (in the case of active mixers). The performances of TPD are better than those of the classic IQ demodulator using two channels shifted by $90^{\circ}$ recovering the baseband IQ signals because it allows a rejection of 20 $\mathrm{dB}$ of signals resulting from intermodulation products of second-order [16]. In fact compared to previous references $[14,15]$ nonlinear effects created by the non-ideal mixers are taking into account in this paper. The demodulator components have been modified in order to deal with bandwidths for each of received carriers more than $10 \mathrm{MHz}$ and thus to increase the throughput of a few hundred kbps to 300 Mbps. The RF performances of the new TPD are described in paper [17]. In this paper the quality of the new TPD will be described by measurement results of phase constellations and EVM of demodulated signals. In references $[12,14,15]$ the separation of the transposed baseband signal is performed by a digital filter. A new numerical method for signal separation is proposed that reduces computation time. Finally, we propose a method to synchronize the six local oscillators of the transmitter and the receiver.

The technique which will be developed in this paper consists of mixing RF signal, constituted of 3 non-contiguous carriers with a LO signal, constituted of 3 $\mathrm{CW}$ signals. Section 2 will present the theory of the transposition in low frequency in two cases: ideal and non-linear mixer. Section 3 will describe the architecture of the TPD and the principle of the data processing. Section 4 will show the experimental results and the principle of phase recovery of RF signal and finally, section 5 will give a conclusion.

\section{Principe of Baseband Conversion}

The aggregated RF signals are down converted to a small band of several tens of megahertz, named "Br: signals recovery band". The narrow bandwidth $\mathrm{Br}$ has a frequency width that covers the spectrum of the baseband signals from zero to $f_{L P F}$ where $f_{L P F}$ is the cutoff frequency of the low-pass filter which will be higher than the sum of the three frequency bandwidths of the RF signal. Let us consider three RF signals centered on three non-contiguous carriers $f_{1}, f_{2}$ and $f_{3}$, respectively. The associated bandwidths of three modulated carriers are $B W_{1}, B W_{2}$ and $B W_{3}$, respectively. The expression of the RF signal is defined as follows

$$
s_{R F}(t)=\sum_{i=1}^{3} \mathfrak{R}_{e}\left(z_{i}(t) e^{j 2 \pi f_{i} t}\right)
$$

with $z_{i}(t)$ is the complex envelope of the three carriers frequencies $f_{1}, f_{2}$ and $f_{3}$.

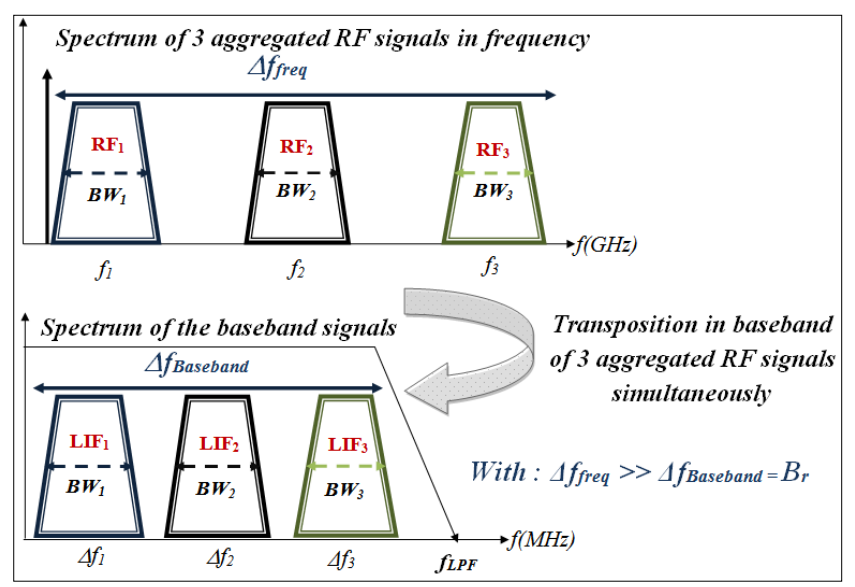

Figure 1. Illustration of down-conversion of the three non-contiguous aggregated carriers modulated

$$
z_{i}(t)=a_{i}(t) e^{j \theta_{i}(t)}=I_{i}(t)+Q_{i}(t) \quad(1 \leq i \leq 3)
$$

The baseband transposition of the aggregated RF signals is depicted in figure 1 . In the case where the LO signal is made 
of a single CW signal, the transposed signal occupies a very wide frequency band which can reach several $\mathrm{GHz}\left(f_{3}-f_{1}\right)$. Separating the shifted signals by a digital processor requires an ADC with a very high sampling frequency. The goal is to translate signals in a band Br slightly greater than the sum of the bandwidths $B W_{i}$ of the carrier signals.

Let us consider the LO signal constituted of $3 \mathrm{CW}$ signal of frequencies : $f_{L O_{i}}=f_{i}-\Delta f_{i}$

$$
S_{L O}(t)=\mathfrak{R}_{e}\left(\sum_{i=1}^{3} V_{L O} e^{j 2 \pi f_{L O_{i}} t}\right)
$$

With the same amplitude $V_{L O}$ for all CW signals.

Two constraints must be taken into consideration at the time of down-conversion. First is to avoid overlapping between the transposed useful signals in baseband. The choice of the intermediate frequency $\Delta f_{i}$ depends on the bandwidth $B W_{i}$ of the $\mathrm{i}^{\text {th }} \mathrm{RF}$ signal.

$$
\Delta f_{j}-\left(B W_{j} / 2\right)>\Delta f_{j-1}+\left(B W_{j-1} / 2\right) \quad(2 \leq j \leq 3)
$$

The second condition is illustrated in the figure 1. We choose the carriers which have a significant gap between them in such a way to have the following condition: $f_{2}-f_{1}>B_{r}$ and $f_{3}-f_{2}>B_{r}$.

So the necessary conditions for the recovery of converted signals in baseband by a single demodulator are summarized as:

$$
\begin{aligned}
& f_{2}-f_{1}>B_{r}, f_{3}-f_{2}>B_{r} \\
& \Delta f_{j}-\left(B W_{j} / 2\right)>\Delta f_{j-1}+\left(B W_{j-1} / 2\right),(2 \leq j \leq 3)
\end{aligned}
$$

\subsection{Ideal Mixer}

Firstly, we consider that the mixers of the demodulator circuit are perfectly linear. At the output of the path $m$ ( $m$ $\in\{3,4,5\})$

$$
v_{m}(t)=\operatorname{LPF}\left\{s_{R F}(t) s_{L O}(t)\right\}
$$

where LPF is the low-pass filter of cutoff frequency equals to $f_{L P F}$.

Assuming the conditions of (5) are fulfilled, we deduce from equation (6):

$$
v_{m}(t)=\sum_{i=1}^{3} V_{L O} a_{i} L_{m i} \cos \left(\left(\theta_{i}+2 \pi \Delta f_{i}\right) t-\varphi_{m i}\right)
$$

With $L_{m i}, \varphi_{m i}$ the conversion loss and the phase shift introduced by the path $m$ of the demodulator at frequency $f_{i}$. The equation (7) shows that the output signal of the path $m$ is the sum of three LIF signals centered at $\Delta f_{1}, \Delta f_{2}$ and $\Delta f_{3}$ respectively. This result confirms the stated hypothesis in this section.

\subsection{Non- Ideal Mixer}

We assume that the mixer is non-ideal: There are the self-mixing of LO and RF signals due to the bad isolation $I s O_{L O}$ and $I s o_{R F}$ between LO and RF ports of the mixer. The mixing process is non-linear and can be developed by $n$ order Taylor series of the product of RF and LO signals. As in reference [18] the non linearities are introduced after the mixing operation. Using these assumptions the output voltage $S(t)$ of the mixer is :

$$
\begin{aligned}
S(t) & =I s O_{L O}\left(s_{L O}\right)^{2}+I s O_{R F}\left(s_{R F}\right)^{2}+L_{m i} s_{R F} s_{L O} \\
& +g_{2}\left(s_{R F} s_{L O}\right)^{2}+g_{3}\left(s_{R F} s_{L O}\right)^{3}
\end{aligned}
$$

where $L_{m i}$ represents the linear term and $g_{2}$ and $g_{3}$ the terms of order 2 and 3 . In general if $n \geq 3$, the terms are negligible because they correspond to signals of low power level.

We consider in the following that $L_{m i}$ is a constant whatever frequency $f_{i}$ and that all three carriers signals of the RF signal have the same amplitude $A$ and are not modulated.

$$
S_{R F}(t)=\sum_{i=1}^{3} A \cos \left(2 \pi f_{i} t\right)
$$

As the input signals are sinusoidal, each term $\cos ^{p}\left(2 \pi f_{i} t\right)$ generates harmonic $p f_{i}$ and each term $\cos ^{p}\left(2 \pi f_{i} t\right) \cos ^{n}\left(2 \pi f_{L O_{j}} t\right)$ generates two frequencies: the sum frequency $\mathrm{p} f_{i}+n f_{L O j}$ and the difference frequency $p f_{i}$ $-n f_{L O j}$ (with $\mathrm{p}$ and $\mathrm{n}$ two positive integers). A non-linear system generates therefore a multiple of frequencies which are harmonics and intermodulation products whose amplitudes decreases when $\mathrm{p}$ and $\mathrm{n}$ increase.

The frequencies of the RF and LO signals are, in general, in the order of several hundreds of megahertz to a few gigahertz. While the signals which will be in the recovery frequency band $B_{r}$ will be of order of tens of megahertz. So during the transposition in low frequency, the harmonic terms into $\mathrm{n} f$ are found in general far useful signal i.e. out of recovery band.

As an example, according to section 4 , the 3 carrier frequencies are $2.9 \mathrm{GHz}, 2.4 \mathrm{GHz}$ and $2.084 \mathrm{GHz}$ and the $\mathrm{LO}$ signal is the combination of $3 \mathrm{CW}$ signals centered at 2.89 $\mathrm{GHz}, 2.352 \mathrm{GHz}$ and $2 \mathrm{GHz}$. The intermediate frequencies of LIF signals are: $\Delta f_{1}=10 \mathrm{MHz}, \Delta f_{2}=48 \mathrm{MHz}$ and $\Delta f_{3}=84 \mathrm{MHz}$ and the cutoff frequency $f_{L P F}$ of the low-pass filter is equal to $100 \mathrm{MHz}$. In that way, the conditions (5) are totally fulfilled, thus after filtering results of (8) equation (10) is obtained. The first term of $v_{m}(t)$ is the useful signal, the others are the spurious signals caused by the bad isolation and the non-linearity of the mixer. In fact expressions are more complex as the complex envelope and the phase shift of LO signal on path $\mathrm{m}$ of the demodulator are not included. In the next section, we will show that the parasitic effects are very low as we can notice on the results of figures 5 and 8 . This is due to the architecture of the demodulator that performs a projection of baseband voltages $v_{m}(t)$ into a I and Q plane 
thus rejecting partially the spurious signals $[19,20]$ and as well to the low values of $g_{2}$ and $g_{3}$.

$$
\begin{aligned}
v_{m}(t)= & L P F(S(t))=\sum_{i=1}^{3} L_{m i} V_{L O} A \cos \left(2 \pi \Delta f_{i} t\right) \\
& +\frac{3 I s o_{L O}\left(V_{L O}\right)^{2}}{2}+\frac{3 I s o_{R F} A^{2}}{2} \\
& +\frac{g_{2} V_{L O}^{2} A^{2}}{18}\left[18+\cos \left(4 \pi \Delta f_{1} t\right) \cos \left(4 \pi \Delta f_{2} t\right)\right] \\
& +\frac{g_{3} V_{L O}^{2} A^{2}}{32}\left[\cos \left(6 \pi \Delta f_{1} t\right)+9 \sum_{i=1}^{3} \cos \left(2 \pi \Delta f_{i} t\right)\right]
\end{aligned}
$$

\section{TPD Architecture and Data Processing}

\subsection{TPD}

Figure 2 shows the full assembly of the implemented demodulation circuit: two 3-way combiners, Anaren-043020, allow the division of $\mathrm{LO}$ and RF signals. The frequency band of the combiner Anaren- 043020 is from 2 to $6 \mathrm{GHz}$. The mixing of signals is realized by active mixers of Meuro Microwave (MMB0050050F0020). The frequency band of mixers varies from 0.45 to $3.6 \mathrm{GHz}$.

The operations described in $[19,21]$ in the case of five-port are also equivalent in the case of the TPD. The low frequency signal $v_{m}(t)$ at the output of the non-ideal mixer $m$, ( $m=3,4$ and 5 ), can be written as follows :

$$
\begin{gathered}
v_{m}(t)=D C_{m}+I M D_{m}(t) \\
+\sum_{i=1}^{3} V_{L O} a_{i} L_{m i}\left[I_{i}(t) \cos \left(\varphi_{m i}\right)+Q_{i}(t) \sin \left(\varphi_{m i}\right)\right], \\
\mathrm{m}=\{3,4,5\}
\end{gathered}
$$

where $I_{i}(t)$ and $Q_{i}(t)$ are the baseband signals in phase and quadrature at frequency $f_{i}$; The signal $v_{m}(t)$ has three terms: The first term is the DC (Direct Current) component that is due essentially to LO signal self-mixing at each $\mathrm{CW}$ frequency $\left(f_{i}-\Delta f_{i}\right)$; the second term results the self-mixing of useful signals and is called the intermodulation distortion (IMD) products(Equation(10)). Another contribution to IMD comes from other adjacent strong signals, separated in frequency an amount $\Delta_{a d j=} f_{a d j 2}-f_{a d j l}$, less than the bandwidth of interest $B_{r}$. And the third term contains the desired $I_{i}(t)$ and $Q_{i}(t)$ signals.

[20] shows that the baseband signals $I_{i}(t)$ and $Q_{i}(t)$ can be found by a projection of 3 voltages $v_{m}(t)$ on a IQ plane. The coefficient of the matrix associated to this projection was determined by a self-calibration procedure. In order to reduce the numerical processing task, the coefficient of the matrix can be predetermined by choosing the architecture of the TPD $[19,21]$. If path number 4 of the TPD is an axis of symmetry compared to the two others, and $L_{m i}$ the conversion loss is identical for the 3 paths of the TPD at frequency $f_{i}[19]$ shows:

$$
\begin{aligned}
& \sum_{i=1}^{3} I_{i}(t)=\mu_{I}\left[2 v_{4}(t)-v_{3}(t)-v_{5}(t)\right]=\mu_{I} I_{\text {out }}(t) \\
& \sum_{i=1}^{3} Q_{i}(t)=\mu_{Q}\left[v_{3}(t)-v_{5}(t)\right]=\mu_{Q} Q_{\text {out }}(t)
\end{aligned}
$$

where

$$
\begin{aligned}
& \mu_{Q}=\tan \left(\frac{\gamma_{i}}{2}\right) \square_{I} \\
& \mu_{I}=\frac{1}{V_{L O} a_{i} L_{m i}} \frac{1}{1-\cos \left(\gamma_{i}\right)}, \\
& \gamma_{i}=\varphi_{3 i}-\varphi_{4 i}=\varphi_{4 i}-\varphi_{5 i} \\
& \mu_{Q}=\tan \left(\frac{\gamma_{i}}{2}\right) \square_{I} \\
& \mu_{I}=\frac{1}{V_{L O} a_{i} L_{m i}} \frac{1}{1-\cos \left(\gamma_{i}\right)}, \\
& \gamma_{i}=\varphi_{3 i}-\varphi_{4 i}=\varphi_{4 i}-\varphi_{5 i}
\end{aligned}
$$




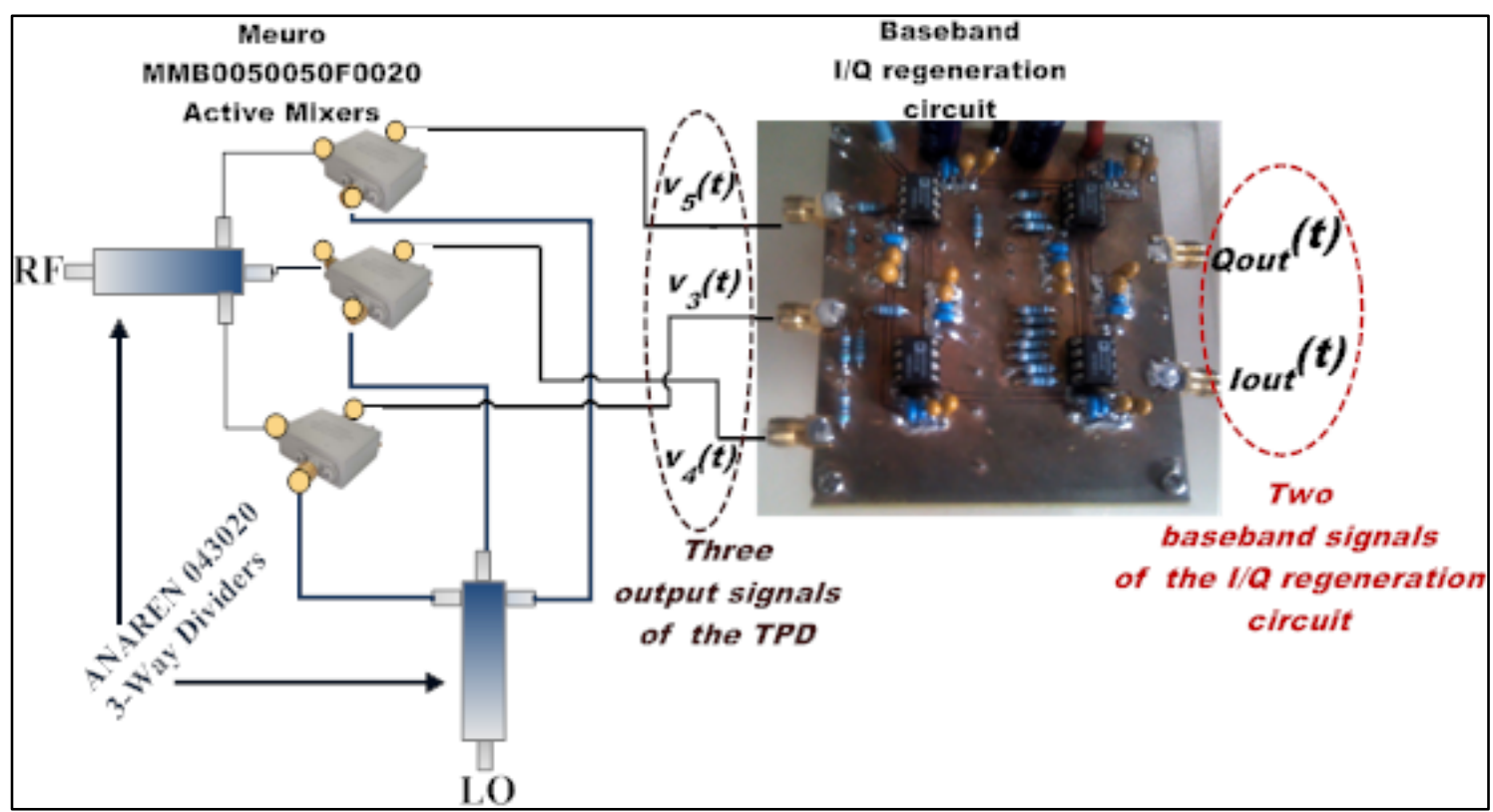

Figure 2. TPD architecture with baseband I/Q regeneration circuit.

The addition of voltages is performed by an analog I/Q regeneration circuit realized by Operational amplifiers of Analog Devices (AD8056). The functioning results of the demodulator are explained in section 5 of [17]. The measurement results of [16], [17] demonstrate that the TPD circuit gives a gain of $20 \mathrm{~dB}$ in IMD2 rejection in comparison with classical IQ circuit. Indeed [19] demonstrates that if conversion losses $L_{m i}$ are equal on the 3 paths of the TPD, there is a perfect cancellation of DC offset and IMD at the output of the regeneration circuit. Morever if $\gamma_{i}=90^{\circ}$ there is a perfect equality between I/Q signals. In addition, the measured EVM of baseband signals resulting by the demodulation of RF signals composed of two non-contiguous carriers are better in TPD circuit than the classic IQ circuit [17]. Therefore, the TPD circuit is presented as a serious candidate for demodulating LTE-A signals. The following section 4 will present the test bench for the demodulation of three non-contiguous modulated carriers.

\subsection{Data Processing}

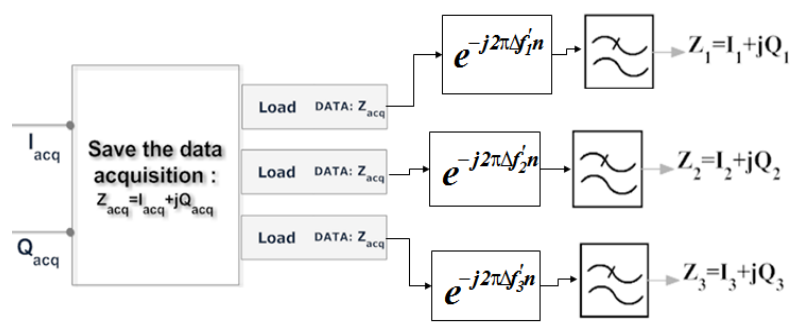

Figure 3. Digital processing of the separation the three baseband signals

The Data processing performs the separation of each LIF signal and their equalization. The first task may be realized using digital filters $[12,14,15]$ consisting of a low-pass filter for the $\mathrm{LIF}_{1}$ signal, a high-pass filter for the $\mathrm{LIF}_{3}$ signal and a pass-band filter for the $\mathrm{LIF}_{2}$ signal. Next, the intermediate frequency is digitally removed for each signal before the equalization. The drawback of this method is its heavy computation. We propose another method to separate of the three baseband signals (Figure 3). After sampling, the data corresponding to the three signals are numerically down-converted and thereafter filtered by a numerical low pass filter. For example, the signal centered at $\Delta f_{l}$, is transposed at zero Hertz by the multiplication of each $n$ data by the complex term $e^{j 2 \pi \Delta f_{1} n}$ (where $\Delta f_{1}^{\prime}=\Delta f_{1} /$ sampling frequency) and then it is applied a low-pass filter of bandwidth higher than the bandwidth of the $\mathrm{LIF}_{1}$ signal. This same process is also applied at $\mathrm{LIF}_{2}$ and $\mathrm{LIF}_{3}$ signals. If the three LIF signal have the same bandwidth, only one LPF digital filter have to be computed thus reducing the computation time.

Each separated signal is equalized by the algorithm MMSE (minimum mean square error) by using a CAZAC (Constant Amplitude Zero Auto Correlation) training sequence. The equalization process compensates the amplitude imbalance of the restored $I_{i}(t)$ and $Q_{i}(t)$ signals by the calculation of $\mu_{I}$ and $\mu_{Q}$.

\section{Experimental Results}

\subsection{Test Bench}

Figure 4 illustrates the measurement bench. 


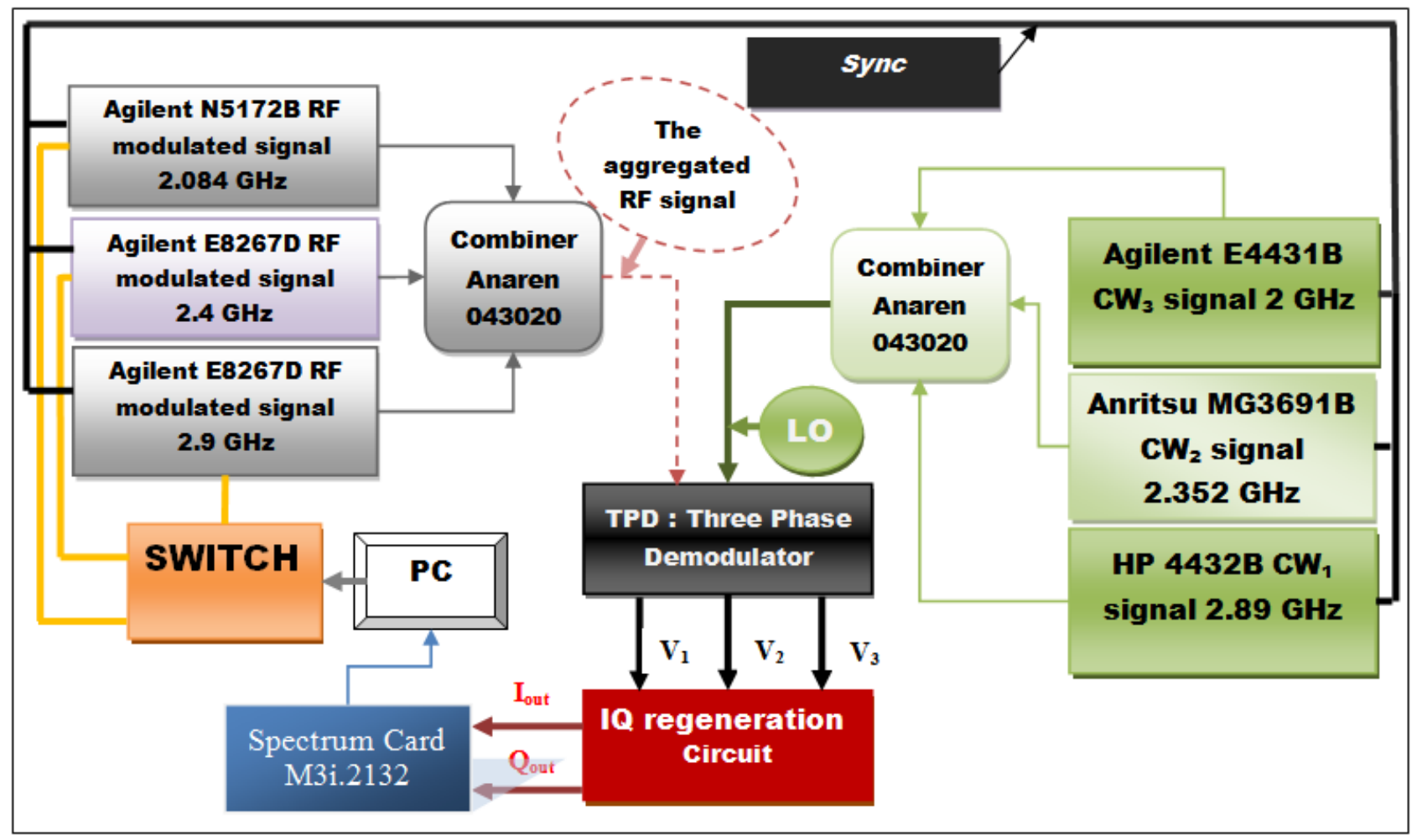

Figure 4. Test bench

From a computer, the digital symbols $/ I(n) /$ and $/ Q(n) /$ are filtered by a square root Nyquist filter (SQRC) of roll-off equal to 0.35 . The filtered frame is composed a 32 bits training sequence of type CAZAC, followed by a Pseudo Noise (PN) data sequence. The training sequence is always encoded in QPSK (Quadrature phase-shift keying). The PN data can be encoded in QPSK or 16-QAM. The frame is then sent to a generator via a LAN network constituted of a Switch of ALLIED TELESIS (AT-FS708). Three different data are uploaded to three generators of Agilent Technologies (E8267D, E8267D and N5172B). The test is performed with a $10 \mathrm{MHz}$ or $15 \mathrm{MHz}$ bandwidth for each modulated carrier frequency. A signal bandwidth of $10 \mathrm{MHz}$ can be demodulated with a data rate of $80 \mathrm{Mbps}$, while the 15 MHz bandwidth is demodulated at $120 \mathrm{Mbps}$ data rate for a 16-QAM modulation. The three RF signals are transmitted at $2.9 \mathrm{GHz}, 2.4 \mathrm{GHz}$ and $2.084 \mathrm{GHz}$. A 3-way combiner, Anaren 043020, adds the three output signals of RF generators. At the output of the combiner, we have a RF signal constituted of three modulated carriers which occupy three non-contiguous bands in the spectrum. This signal will be mixed with a LO signal which is the combination of three $\mathrm{CW}$ signals. According the theoretical principle presented in section 2, the three aggregated RF signals must be transposed in baseband at LIF. The bandwidth of each modulated signal is $10 \mathrm{MHz}$ or $15 \mathrm{MHz}$. Therefore, a recovery band of signals of bandwidth equal to $100 \mathrm{MHz}$ and a choice of intermediate frequencies of $10 \mathrm{MHz}, 48 \mathrm{MHz}$ (or $40 \mathrm{MHz}$ ) and $84 \mathrm{MHz}$ fulfill the conditions of (5). Hence the LO signal is the combination of $3 \mathrm{CW}$ signals centered at $2.89 \mathrm{GHz}, 2.352$ $\mathrm{GHz}$ (or $2.360 \mathrm{GHz}$, if the intermediate frequency is equal to $40 \mathrm{MHz}$ ) and $2 \mathrm{GHz}$, respectively.

The output signals of 3 sources (HP 4432B, Anritsu MG3691B and Agilent E4431B) are combined to generate the three $\mathrm{CW}$ signals at $0 \mathrm{dBm}$. All sources are synchronized by using the same clock signal at frequency 10 MHz. However in [22] a method to generate simultaneously these three $\mathrm{CW}$ signals by a single generator is explained.

The LO and RF signals are applied at the inputs of the TPD and the output signals of the TPD are injected into the analog I/Q regeneration circuit. The output signals $\mathrm{I}_{\text {out }}$ and $\mathrm{Q}_{\text {out }}$ are sampled at $240 \mathrm{MHz}$ via a data acquisition card (SPECTRUM M31.2132). The acquisition card can reach a maximum sampling rate of 500 MSamples/s for two channels.

\subsection{Spectrum, phase constellations and EVM results}

Figure 5 shows the measured spectrum at the output of $\mathrm{I}_{\text {out }}$ path by FSIQ40 Rhode \& Schwartz (spectrum analyzer). Three signals of $10 \mathrm{MHz}$ bandwidth, each, are used. The LIF signals are centered at intermediate frequencies of $10 \mathrm{MHz}$, $40 \mathrm{MHz}$ and $84 \mathrm{MHz}$. Each LIF signal occupies a wide bandwidth of $13.5 \mathrm{MHz}$ (let $2 * 6.75 \mathrm{MHz}$ ). This result corresponds to the bandwidth of the digital data filter at the transmission. The spectrum of three LIF signals shown in Figure 5 confirms the theoretical principle of section 2 . 


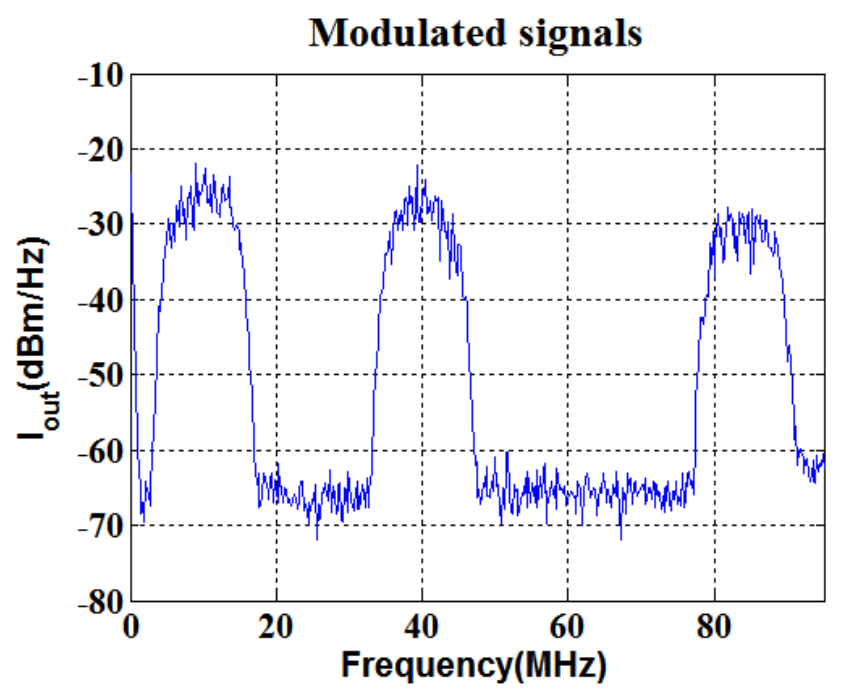

Figure 5. Measured baseband spectrum at the output of the I/Q regeneration circuit of Iout path; the input power level is equal to $-25 \mathrm{dBm}$.

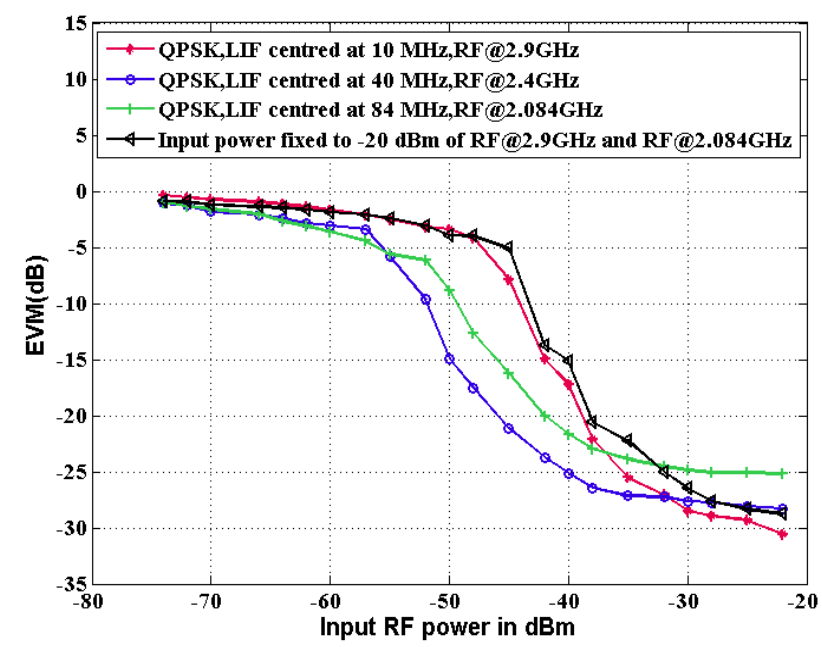

Figure 6. Measured EVMs of different demodulated LIF signals in function of the RF input power level; all signals have the same bandwidth of $10 \mathrm{MHz}$.

Figure 6 shows the measured EVM of different demodulated LIF signals in function of the RF input power level;. The deterioration of the centered LIF signal at 10 $\mathrm{MHz}$ when the RF input power is less than $-35 \mathrm{dBm}$ can be explained by the presence of significant parasitic effects at frequencies that are close to zero frequency. The centered LIF signal at $84 \mathrm{MHz}$ has the worst EVM for RF input power above $-35 \mathrm{dBm}$. Also, one may observe that EVM of this signal is 3 to $5 \mathrm{~dB}$ worse than EVM of the centered LIF signal at $40 \mathrm{MHz}$ for input power of $-55 \mathrm{dBm}$ to $-35 \mathrm{dBm}$. This performance degradation at $84 \mathrm{MHz}$ may be due to the clock jitter induced in the sampling of the highest LIF signal [23]. Another experiment has been conducted to take into account channel fading and channel loss at different carrier frequencies. The curve in black color, Figure 6, shows the variation of EVM of demodulated symbols in function of input power level of RF signal centered to $2.4 \mathrm{GHz}$, keeping constant signal power levels to $-20 \mathrm{dBm}$ in the other two frequency bands 2.084 and $2.9 \mathrm{GHz}$. Fixing the value of EVM to $-15 \mathrm{~dB}$, the required power level of the signal centered at $2.4 \mathrm{GHz}$ is increasing from $-50 \mathrm{dBm}$ to $-40 \mathrm{dBm}$. This effect is due to the power level differences of 3 LIF signals. It may be corrected by using different power levels for each of the CW signal of the LO [11].

In the following, the RF signals are transposed at intermediate frequencies of $10 \mathrm{MHz}, 48 \mathrm{MHz}$ and $84 \mathrm{MHz}$, respectively. The intermediate frequency of the second signal is moved from $40 \mathrm{MHz}$ to $48 \mathrm{MHz}$. The following results prove that the signal centered at $40 \mathrm{MHz}(4 * 10 \mathrm{MHz})$ is not an image of the centered signal at $10 \mathrm{MHz}$.

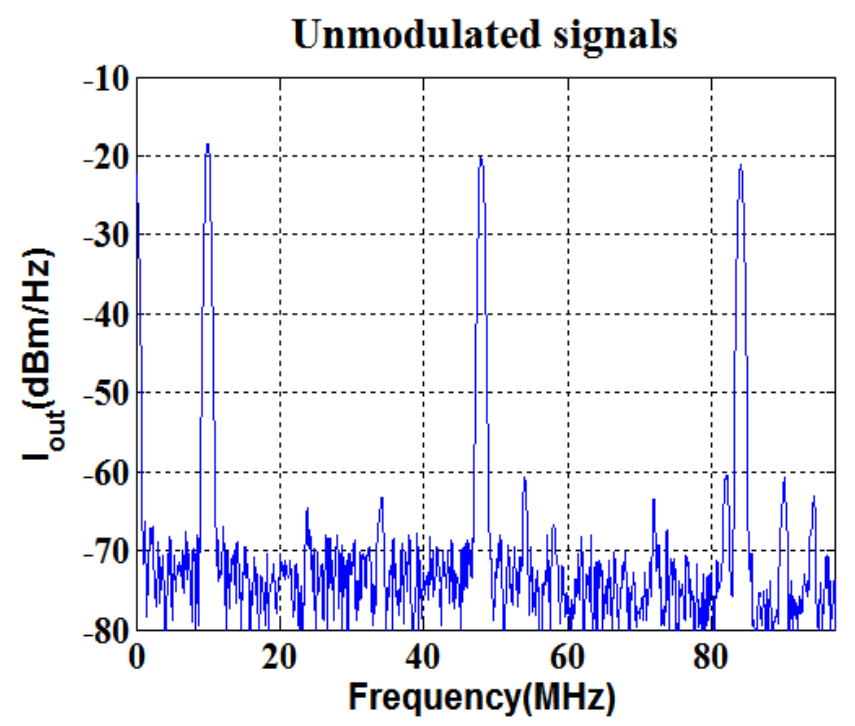

(a)

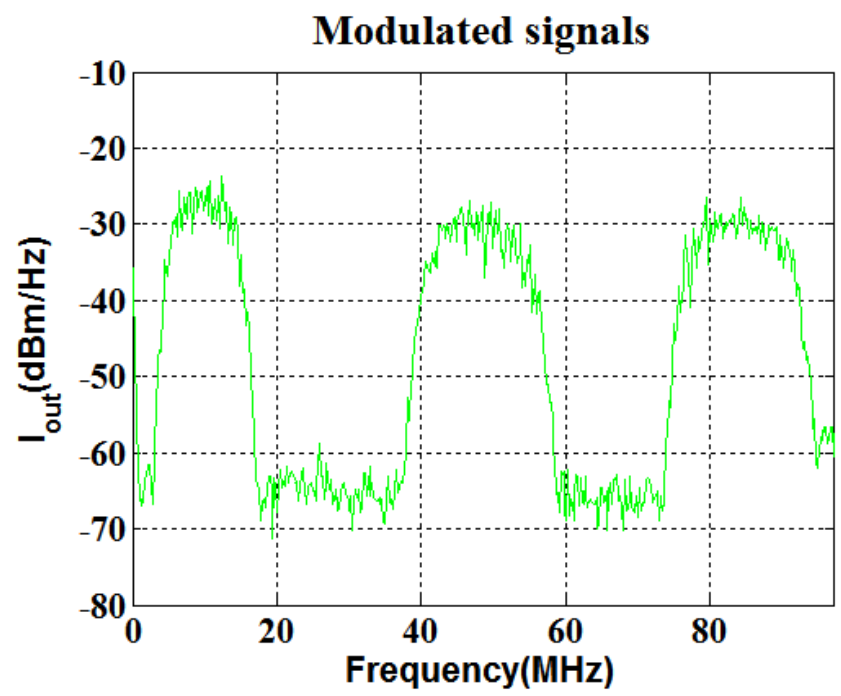

(b)

Figure 7. Measured baseband spectrum at the output of $I_{\text {out }}$ path of the $\mathrm{I} / \mathrm{Q}$ analog regeneration circuit, the RF input power level is equal to -25 $\mathrm{dBm}$; (a) unmodulated signals; (b) three modulated LIF signals of bandwidths $10 \mathrm{MHz}, 15 \mathrm{MHz}$ and $15 \mathrm{MHz}$

Figure 7 shows the measured spectra at the output of $\mathrm{I}_{\text {out }}$ in the case of an input power level of $-25 \mathrm{dBm}$. Figure 7(a) presents the measured spectrums of three unmodulated 
transposed carriers. The levels of the spurious signals are less than $-60 \mathrm{dBm}$, so they are sufficiently low compared to the useful signals. There is a difference of about $40 \mathrm{~dB}$ between the useful and spurious signals.

Figure 7(b) shows the spectrum of three modulated carriers when they are transposed in low frequency with bandwidths of $10 \mathrm{MHz}, 15 \mathrm{MHz}$ and $15 \mathrm{MHz}$, respectively. The spectrum of the $\mathrm{LIF}_{1}$ signal of the Figure 7(b) occupies about a bandwidth of $13.5 \mathrm{MHz}$, the two centered at $48 \mathrm{MHz}$ and $84 \mathrm{MHz}$ occupy each a bandwidth of about $20.25 \mathrm{MHz}$ (let $2 * 10.125 \mathrm{MHz}$ ). The results of figures $7(\mathrm{a})$ and $7(\mathrm{~b})$ confirm again the theoretical principle of section 2 .

Figure 8 presents the measured constellations diagrams in the case where the modulated carriers have bandwidths of 10 $\mathrm{MHz}, 15 \mathrm{MHz}$ and $15 \mathrm{MHz}$, respectively. Figure 8(a) illustrates the constellations where all aggregated signals in frequency are modulated in QPSK. Figure 8(b) shows the constellations for two different modulation formats (QPSK and 16-QAM).
Figure 9 shows the measured EVMs in function of the input power level. The results of figure 9 present the case where we have two different bandwidths for the frequency aggregated signals. The remarks made above (the results of the Figure 6) remain valid for results of Figure 9(a). By comparing the measured EVM of the centered signal at 40 $\mathrm{MHz}$ of the figure 6 and that of the centered signal at $48 \mathrm{MHz}$ of the figure 9(a), we note that in figure 9(a) an attenuation of 6 to $10 \mathrm{~dB}$ with respect to Figure 6 can be observed for a variation of the input power level from $-55 \mathrm{dBm}$ to $-40 \mathrm{dBm}$. This attenuation can be explained by the difference of the Over-Sampling Ratio (OSR) between the two signals. In fact, as we used the same sampling frequency equal to $240 \mathrm{MHz}$, it is clear that the signal of $10 \mathrm{MHz}$ bandwidth has an OSR equal to 24 and it is equal to 16 for the signal of bandwidth to $15 \mathrm{MHz}$. The measured EVMs of the figure 9(b) show that the QPSK modulation format is more robust than 16QAM modulation format.

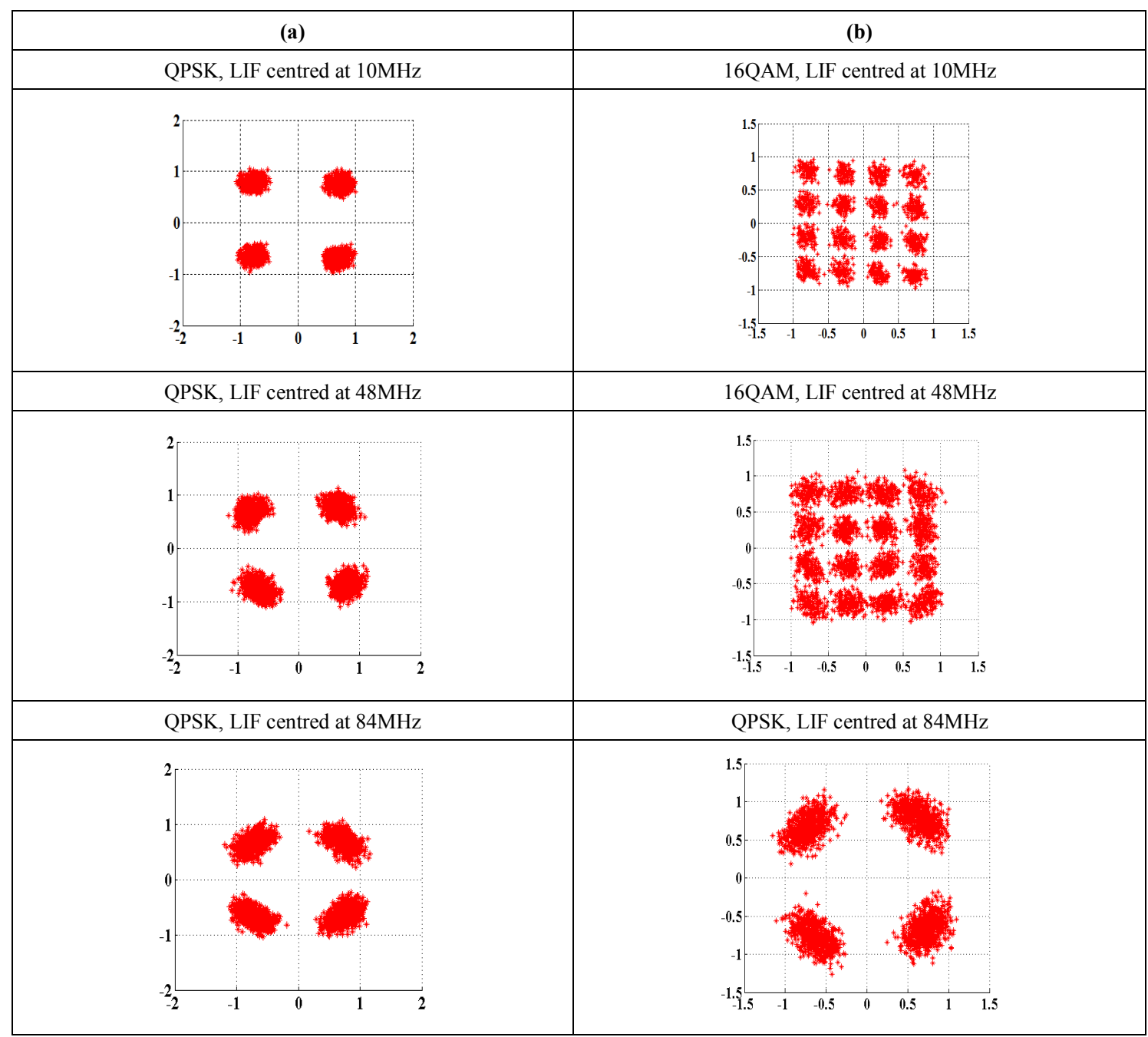

Figure 8. Measured constellations diagrams at the input power level equal to $-25 \mathrm{dBm}$ (a) three QPSK-modulations (b) two 16-QAM modulations and one QPSK modulation. 


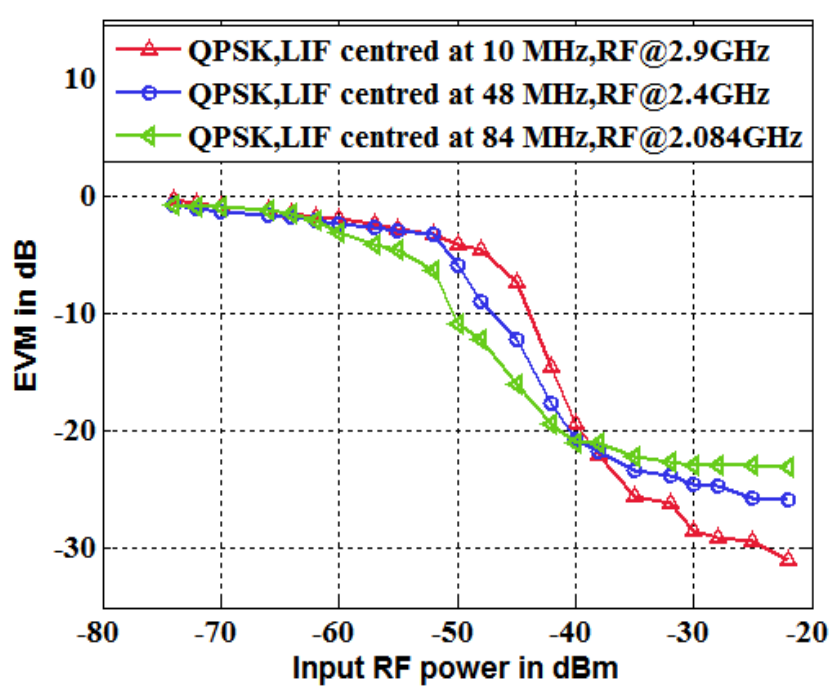

(a)

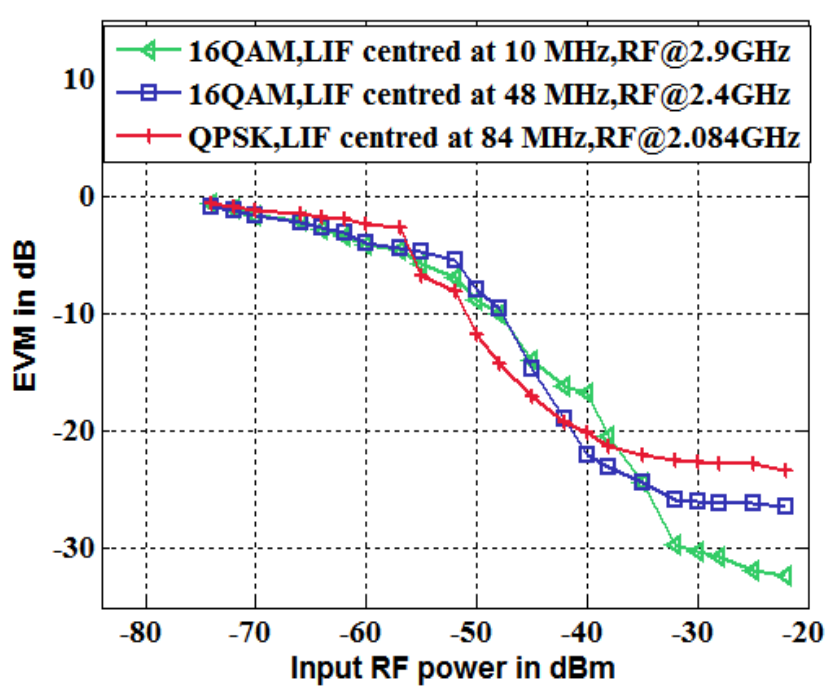

(b)

Figure 9. Measured EVMs of different demodulated LIF signals in function of the RF input power level (a) three QPSK-modulations (b) two different modulations format.

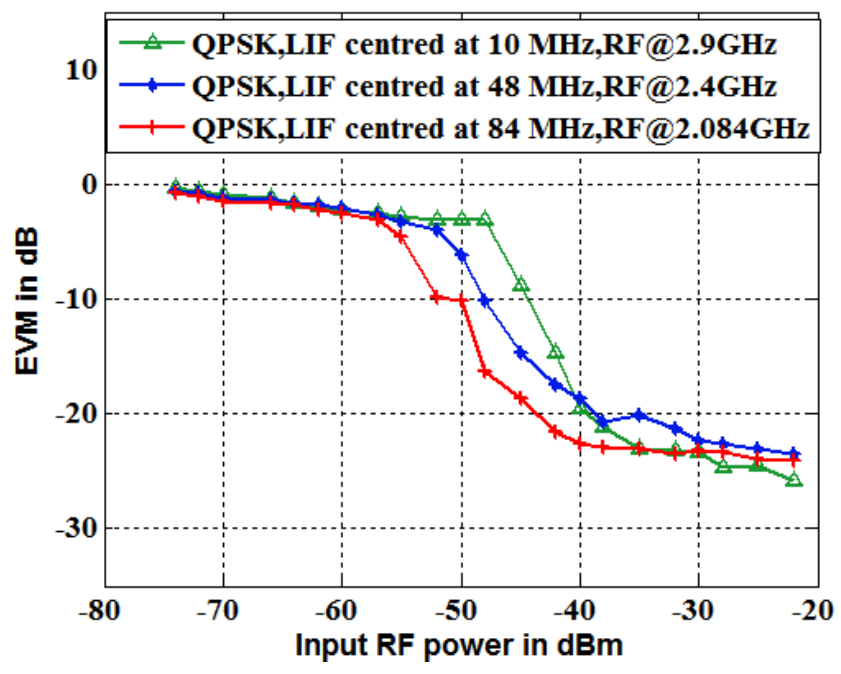

Figure 10. Measured EVMs of different demodulated LIF signals in function the RF input power level; all signals are the same bandwidth of 15 $\mathrm{MHz}$.

Figure 10 shows the measured EVMs in the case where the frequency aggregated signals have the same bandwidth of 15 $\mathrm{MHz}$. We have an EVM less than $-15 \mathrm{~dB}$ from the RF input power level higher than to $-42 \mathrm{dBm}$ for the centered signal at $10 \mathrm{MHz}$; while it is less than $-15 \mathrm{~dB}$ from the RF input power level higher than $-45 \mathrm{dBm}$ for the centered signal at $48 \mathrm{MHz}$; and it is less than $-15 \mathrm{~dB}$ from the RF input power higher than $-47 \mathrm{dBm}$ for the centered signal at $84 \mathrm{MHz}$. We have a difference which varies from 1 to $3 \mathrm{~dB}$ between adjacent LIF signals. The degradation is more important on the centered signal at $10 \mathrm{MHz}$. The poor quality of the centered signal at
$10 \mathrm{MHz}$ can be caused by spurious effects close to zero frequency. By looking at the result of figure 9-(a), the conclusion is that the increase of bandwidth of the transposed signal at intermediate frequency of $10 \mathrm{MHz}$ has also affected the quality of this demodulated signal.

\subsection{Phase Recovery of Carriers}

The method which will be applied here is essentially based on the one developed in [24], section 4.3.3, for the case of a RF signal constituted of a single frequency carrier. Previously, the generators at the transmitter and the receiver were synchronized from the same clock signal at frequency $10 \mathrm{MHz}$, which does not correspond to the real situation of a wireless link. Figure 11 depicts the test bench which is equivalent to that of Fig. 4 except that is no wired connection between the sources of the transmitter and the receiver. We consider that the carriers of the modulated RF signal of the transmitter are synchronized. Similarly the CW signals of the LO signal are synchronized. The objective is to synchronize the carriers of the aggregated signal and the LO signal of the receiver i.e. to determine the frequency error between the transmitter (Tx) and the receiver ( $\mathrm{Rx})$.

The recovery principle of frequency offset is described as follows: The data frame which will be sent to RF sources is different than that described in subsection 4.1. It is composed of two consecutive CAZAC sequences each with a length of 32 bits followed by a sequence data. 


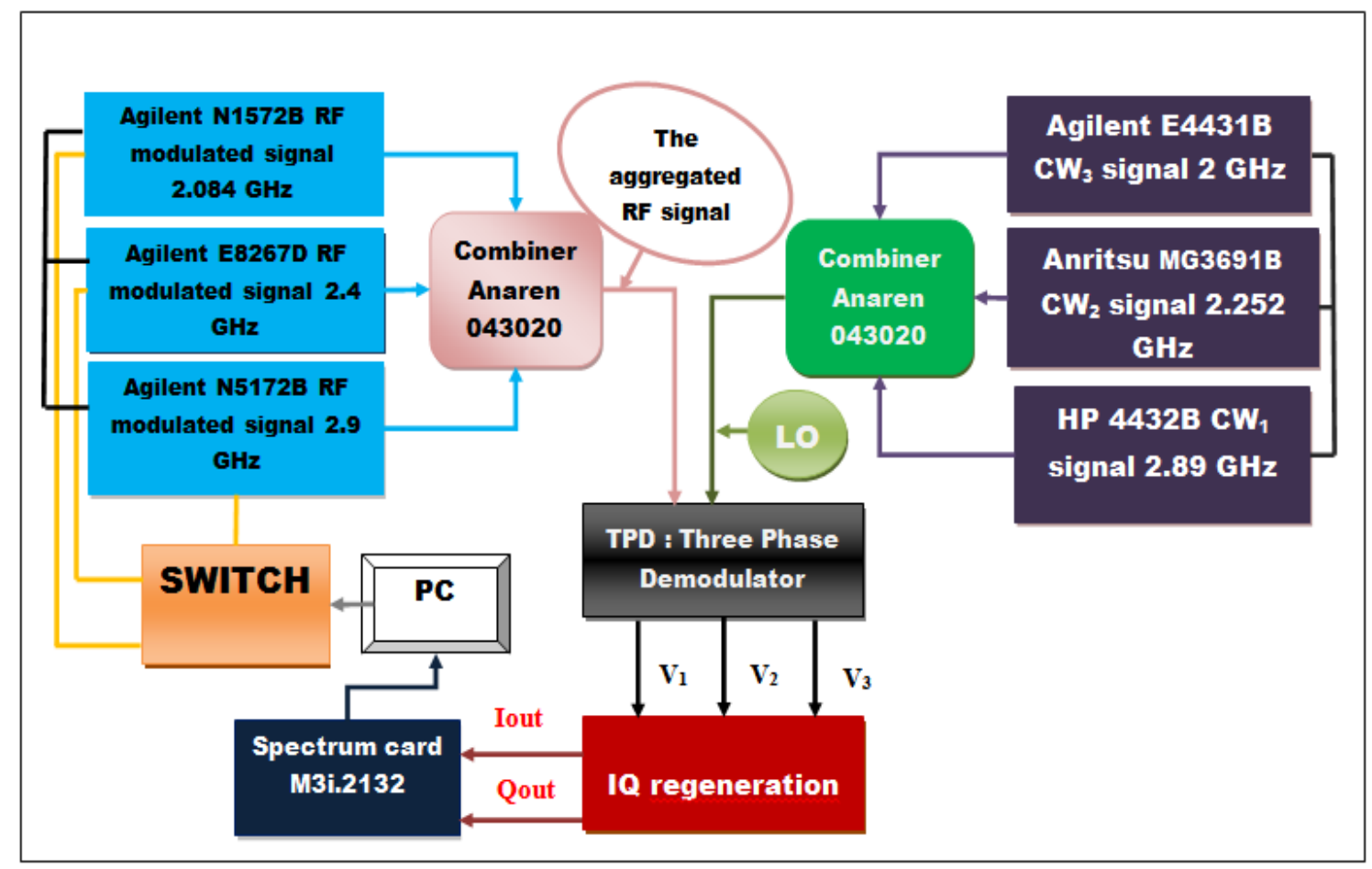

Figure 11. Bench for the recovery of the frequency error between the transmitter and the receiver.

Thus, the new training sequence (TS) has a length of 64 bits. The two CAZAC sequences contain 16 symbols each, because the modulation of the TS sequence is QPSK format. Then, we have two consecutive identical sequence of same size $T S=\left[\begin{array}{ll}T S_{1} & T S_{2}\end{array}\right]$. In other words, we have:

$$
\begin{gathered}
{\left[\mathrm{I}_{\mathrm{TS} 1}(1), \ldots, \mathrm{I}_{\mathrm{TS} 1}(16)\right]=\left[\mathrm{I}_{\mathrm{TS} 2}(1), \ldots, \mathrm{I}_{\mathrm{TS} 2}(16)\right] \&} \\
{\left[Q_{T S 1}(1), \ldots, Q_{T S 1}(16)\right]=\left[Q_{T S 2}(1), \ldots, Q_{T S 2}(16)\right]}
\end{gathered}
$$

The new frame is shown in figure 12.

\begin{tabular}{|c|c|c|}
\hline $\begin{array}{c}\mathrm{TS}_{1}: \\
16 \text { symbols }\end{array}$ & $\begin{array}{c}\mathrm{TS}_{2}: \\
16 \text { symbols }\end{array}$ & DATA \\
\hline
\end{tabular}

Figure 12. The new frame of distributed data to the sources of RF signals.

The CAZAC training sequence allows finding the beginning of information data at the moment of reception. This sequence is known at the transmitter and the receiver. Thus, it permits the sampling optimal time for the frame and symbol synchronization. This sequence will serve us also to make the synchronization of the carrier frequency. This method is done digitally. The frequency offset between the transmitter and the receiver generates a phase error. Therefore, the demodulated signal will rotate with an angular velocity $\theta$. Our purpose is to estimate and cancel $\theta$.. The frequency offset to find is the phase shift between the first symbol and the $17^{\text {th }}$ of training sequence, of the second symbol and the $18^{\text {th }}$ symbol and so on. Figure 13 illustrates the recovery principle of the frequency offset. We note $\mathrm{T}_{\text {sym }}$ the symbol duration and $\Delta f$ the frequency offset to determine.

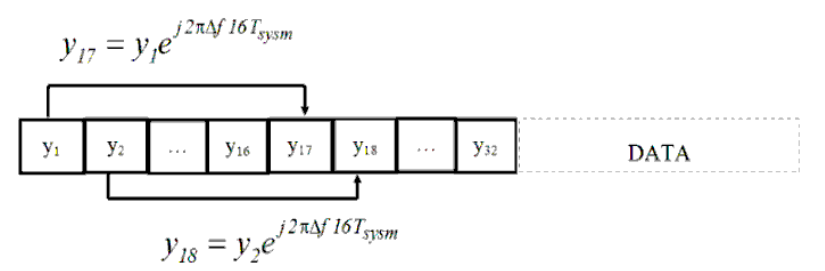

Figure 13. Procedure for estimating the frequency error using the training sequence

The angular velocity of the rotation of the first symbol of $\mathrm{TS}_{2}$ in the phase constellation will almost be equal to that of the first symbol of $\mathrm{TS}_{1}$ during the reception. We denote the duration of 16 symbols, $\Delta T=16 T$ sym.

Thus, we can deduce the following expression:

$$
y_{k+16}=y_{k} e^{j 2 \pi \Delta f \Delta T} \text {, with } 1 \leq k \leq 16
$$

As the symbols of TS are complex elements, only the argument of each of multiplications of (15) needs to be calculated. This operation will permit to have an estimated value on the frequency offset.

$$
y_{k+16} \cdot y_{k}^{*}=\left|y_{k}\right|^{2} e^{j 2 \pi \Delta f \Delta T}
$$

Thus we calculate directly the common angle between the symbols and we deduce the frequency error.

$$
\theta=\arg \left(\sum_{k} y_{k+16} \cdot y_{k}^{*}\right), \text { hence: } \Delta \hat{f}=\frac{\theta}{2 \pi \Delta T}
$$

After implementation of the above algorithm, the phase 
error can be cancelled out using the estimated phase. We present the measurement results of demodulation of the three frequency aggregated signals with the same QPSK-modulation. The three signals have the same bandwidth that is equal to $10 \mathrm{MHz}$.

Figure 14(a) shows the received constellations before the estimation and correction the frequency error. All three constellations rotate with unknown angular velocity. The explained method above allowed estimating the frequency error and canceling it. Figure 14(b) shows the demodulated constellations after estimation and correction of the frequency error between the transmitter and the receiver. The estimated error is in the order of $762 \mathrm{~Hz}$ with a RF input power level of $-25 \mathrm{dBm}$. The constellations are synchronized and normalized, which validates the proposed method for the synchronization of the sources at the Tx and Rx.

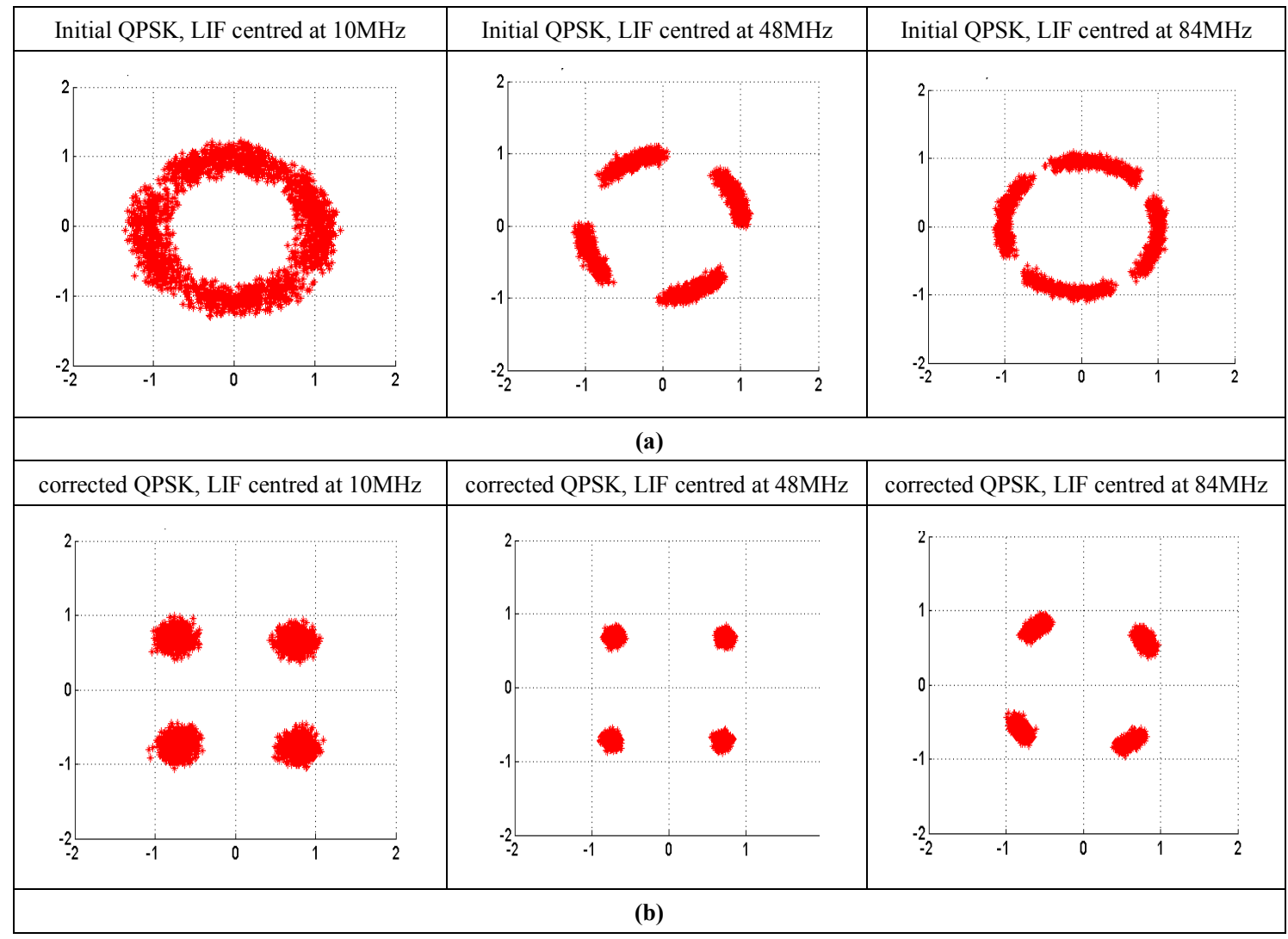

$\Delta \mathrm{f}($ estimated $)=762 \mathrm{~Hz}$

Figure 14. The synchronization between the transmitter and the receiver sources (a) The demodulated signals without correction of the frequency error (b) The constellations of demodulated signals after the correction of the frequency error between the transmitter and the receiver. 
Then, in order to evaluate the maximum frequency error that this technique can recover, the same $10 \mathrm{MHz}$ reference is used for the transmitter and the receiver as explained in subsection 4.1. First each initial carrier frequency of 3 sources is shifted of value $4 \mathrm{kHz}$ but the $3 \mathrm{CW}$ frequencies of LO signal are similar to those of the test bench described in subsection 4.1. Therefore the frequency error is $4 \mathrm{kHz}$. The estimated value during the synchronization is equal to 3.989 $\mathrm{kHz}$. Hence this result confirms again the synchronization method.

Table 1. Test of the algorithm for recovering the frequency difference between the transmitter and the receiver.

\begin{tabular}{|c|c|c|}
\hline $\begin{array}{c}\text { Introduced } \\
\text { frequency error }\end{array}$ & $\begin{array}{c}\text { Estimated } \\
\text { frequency error }\end{array}$ & $\begin{array}{c}\text { Evaluation of the } \\
\text { estimate }\end{array}$ \\
\hline $10 \mathrm{kHz}$ & $10.04 \mathrm{kHz}$ & $\begin{array}{c}\text { Algorithm } \\
\text { succeeded }\end{array}$ \\
\hline $50 \mathrm{kHz}$ & $49.92 \mathrm{kHz}$ & $\begin{array}{c}\text { Algorithm } \\
\text { succeeded }\end{array}$ \\
\hline $100 \mathrm{kHz}$ & $100.86 \mathrm{kHz}$ & $\begin{array}{c}\text { Algorithm } \\
\text { succeeded }\end{array}$ \\
\hline $200 \mathrm{kHz}$ & $199.93 \mathrm{kHz}$ & $\begin{array}{c}\text { Algorithm } \\
\text { succeeded }\end{array}$ \\
\hline $220 \mathrm{kHz}$ & $303.52 \mathrm{kHz}$ & Algorithm failed \\
\hline $300 \mathrm{kHz}$ & $238.28 \mathrm{kHz}$ & Algorithm failed \\
\hline
\end{tabular}

Table 1 presents the estimated values of the frequency difference between the transmitter and the receiver after having introduced a frequency error at the transmission. These tests are performed for an input power level of $-25 \mathrm{dBm}$. The results of Table I show that beyond a frequency difference greater than $200 \mathrm{kHz}$, the algorithm doesn't estimate the desired frequency error.

\section{Conclusions}

This paper has presented a down-conversion technique of a RF signal constituted of three non-contiguous carriers by a single demodulator architecture. The down-conversion of non-contiguous modulated carriers is based on a mixing with a LO signal constituted of $\mathrm{n} \mathrm{CW}$ signals. The tones are chosen to avoid the spurious signals in the recovery band of the useful signals. This method allows reducing the conversion band of ADC. In fact, the RF spectrum was constituted of three non-contiguous frequency bands distributed in the band $2-3 \mathrm{GHz}$ and the transposed signals in baseband occupy a $100 \mathrm{MHz}$ bandwidth. The performances of the TPD on the rejection of unwanted signals such as IMD2 with respect classic IQ architectures, allowed choosing the TPD receiver for the test of the proposed down-converter technique in this paper. The results of obtained spectrums at the output of the demodulator circuit validate the theoretical principle. The measured constellations and EVMs show that it is possible to demodulate multiple frequency aggregated RF signals by a single TPD circuit. The results of the recovery of frequency error between the transmission and the reception sources consolidate the technique of proposed demodulation. The demodulation of three modulated signals in 16-QAM whose each have a $15 \mathrm{MHz}$ bandwidth allows to have a bit rate of about 360 Mbps. So the proposed solution in this paper must to achieve the bit rate of the order of Gigabit in the future mobile network LTE-A.

\section{REFERENCES}

[1] Recommendation ITU-R M.2012-1, "Detailed specifications of the terrestrial radio interfaces of International Mobile Telecommunications-Advanced(IMT-Advanced),"Electronic Publication, Geneva, February 2014.

[2] P. Bhat, S. Nagata, L. Campoy, I. Berberana, T. Derham, L. Guangyi S. Xiaodong, Z. Pingping, Y. Jin, "LTE-advanced: an operator perspective," Communications Magazine, IEEE, vol.50, no.2, pp.104-114, February 2012.

[3] Wannstrom, Jeanette. "LTE-advanced," Third Generation Partnership Project (3GPP) (June 2013). Available at www.3gpp.org.

[4] 3GPP, "Carrier Aggrégation for LTE V0.0.9," Mach 2014, Available at: www.3gpp.org-/ftp/information/work_plan/des cription_releases/

[5] T. Kitayabu, H. Ishikawa, "Implementation of concurrent dual-band receiver using IF undersampling," Radio and Wireless Symposium (RWS), IEEE, Phoenix, AZ, pp.243-246,16-19 Jan.2011.

[6] T. Kitayabu, Y. Ikeda, Y. Amano, H. Ishikawa, "Concurrent dual-band receiver for spectrum aggregation system," Radio and Wireless Symposium, 2009. RWS '09. IEEE, San Diego, CA, pp.634-637, 18-22 Jan. 2009.

[7] L. Sundstrom, S. Ek, J. Svensson, M. Anderson, R. Strandberg, F. Mu, I. ud Din, T. Olsson, L. Wilhelmsson, D. Eckerbert, "Complex IF harmonic rejection mixer for non-contiguous dual carrier reception in $65 \mathrm{~nm}$ CMOS, " IEEE Journal of Solid-State Circuits, vol.48, no.7, pp.1659-1668, July 2013.

[8] L. Sundstrom, M. Anderson, R. Strandberg, S. Ek, J. Svensson, Fenghao Mu, T. Olsson, I. ud Din, L.Wilhelmsson, D. Eckerbert, S. Mattisson, "A receiver for LTE Rel-11 and beyond supporting non-contiguous carrier aggregation," Solid-State Circuits Conference Digest of Technical Papers (ISSCC), 2013 IEEE International, San Francisco, CA, pp.336-337, 17-21 Feb. 2013.

[9] C.S. Park, L. Sundström, A.Wallen, A. Khayrallah,"Carrier aggregation for LTE-advanced: design challenges of terminals," Communications Magazine, IEEE, vol.51, no.12, pp.76-84, Dec. 2013.

[10] L. Chang-Ming, J. Je-Kuan, W. Ping-Hsun, C. Chun-Hsiang, T. Chia-Hao, L. Jian-Yu, "CMOS RF T/R router switch ICs for LTE carrier aggregation transceivers, " Conf. EuMC, 2012 42nd European, Amsterdam, Netherlands, pp.904-907, 29 Oct. 2012- 1 Nov. 2012.

[11] T. Kaho, Y. Yamaguchi, H. Shiba, K. Akabane, K. Uehara, K. Araki, "A simultaneous receiving multi-band mixer with independent gain control," Microwave Conference Proceedings(APMC), 2011, Melbourne, VIC, pp. 383-386, 5-8 Dec. 2011. 
[12] A.O. Olopade, A. Hasan, M. Helaoui, "Concurrent dual-band six-port receiver for multi-standard and software defined radio applications," Microwave Theory and Techniques, IEEE Transactions on, vol.61, no.12, pp.4252-4261, Dec. 2013.

[13] G. Neveux, B. Huyart, J. Rodriguez Guisantes, "Noise figure of a five-port system," In proceedings of the European Conference on Wireless Technology (RCWT '02), Milan, Italy, Sept. 2002.

[14] A. Kaissoine, B. Huyart, A. Mbaye, K. Mabrouk, "Demodulation of aggregated RF signals with a unique Rx chain," New Circuits and Systems Conference (NEWCAS), 2013 IEEE 11th International, Paris, France, pp.1-4, 16-19 June 2013

[15] A. Kaissoine, B. Huyart, K. Mabrouk, "Demodulation of aggregated RF signal in three frequencies bands with a unique $\mathrm{Rx}$ chain," Conf. EuMC, 2013, Nuremberg, Germany, pp.561-564, 6-10 Oct. 2013.

[16] K. Mabrouk, F. Rangel, B. Huyart and G. Neveux, "Architectural solution for second-order intermodulation intercept point improvement in direct down-conversion receivers," IET Microw. Antennas Propag., vol. 4, no. 9, pp. 1377-1386, September 2010.

[17] A. Kaissoine, K. Antoine, K. Mabrouk, B. Huyart, "Performance of $2-3.6 \mathrm{GHz}$ five-port/three-phase demodulators with baseband analog I/Q regeneration circuit in direct-conversion receivers," Hindawi Journal of Electrical and Computer Engineering, vol. 2014, Article ID 631867, 2014.

[18] K. Vandermot, W. Van Moer, J. Schoukens, and Y. Rolain, "Understanding the nonlinearity of a mixer using multisine excitations," ARFTG Conference, pp. 181 - 185, Jun. 2006

[19] C. de la Morena-Álvarez-Palencia, K. Mabrouk, B. Huyart, A. Mbaye, M. Burgos-García: "Direct baseband I-Q regeneration method for five-port receivers improving dc-offset and second-order intermodulation distortion rejection," IEEE Trans. on Microwave Theory \& Techniques, vol. 60, no. 8, pp. 2634-2643, Aug. 2012.

[20] Mabrouk, K.; Huyart, B.; Neveux, G., "3-D aspect in the five-port technique for zero-IF receivers and a new blind calibration method", Microwave Theory and Techniques, IEEE Transactions on, vol.56, no.6, pp.1389,1396, June 2008.

[21] K. Mabrouk, B. Huyart, "Circuit analogique pour le calibrage large bande, la suppression des tensions parasites dues aux dc-offset et produits d'intermodulation IMD2 et réduction d'un convertisseur CAN pour les démodulateurs zéro-if ou low-if de type cinq-port et triphasé", Patent FR2934934 (A1), 2012.

[22] M.Abdi. Abyaneh, A. Kaissoine, B. Huyart, J.C. Cousin, " Multiple RF continuous-wave generation using a single signal generator for carrier aggregation in LTE-advanced," Microwave Conference (EuMC), 2014 European, Rome Italy, 5-10 Oct. 2014,

[23] C. Azeredo-Leme, "Clock jitter effects on sampling: a tutorial," Circuits and Systems Magazine, IEEE, vol.11, no.3, pp.26,37, third quarter 2011.

[24] S. Abou Chakra, " La Boucle Locale Radio et la Démodulation directe de signaux de larges bandes à $26 \mathrm{GHz}, " P h D$ dissertation, Télécom-ParisTech, Paris,France, 20 December 2004. Available at www.pastel.archives-ouvertes.fr. 